Military Technical College Kobry El-Kobbah, Cairo, Egypt

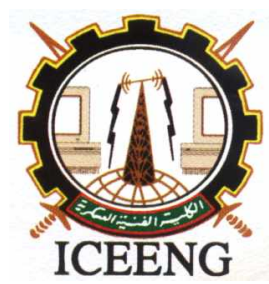

\author{
$6^{\text {th }}$ International Conference \\ on Electrical Engineering \\ ICEENG 2008
}

\title{
CW Radio Seeker Performance Analysis in Presence of Uncertainties for Semi-Active Homing Guidance System
}

By
K. H. Moustfa
Y. Z. Elhalwagy
H. M. Salem

\section{$\underline{\text { Abstract: }}$}

Radio seeker in a semi-active homing guided missile system is a crucial subsystem for performance analysis and development. The signal at the front and rear receivers are mixed to shift the carrier frequency to an intermediate frequency (IF) where the target's Doppler shift can be detected. Such a system has various sources of uncertainties that may significantly affect the radio seeker ability to detect a target correctly including noise, clutter, jamming, and target maneuvers, etc. Uncertainties may lead to degrade the performance of a CW Doppler Monopulse seeker. This paper investigates a semiactive homing missile guidance and control system in presence of jamming, noise and uncertain target maneuvers. In particular, the paper introduces an Electronic Counter Counter-measurements (ECCM) technique that degrades the effectiveness of blinking jamming (BJ) and multiple blinking jamming (MBJ) techniques. These two selfscreening ECM techniques are sources of incoherent deception, where they attempt to attack the tracking dynamics of the angle-tracking radar and, hence, may be effective against some types of Monopulse radar as well as other tracking radars. A mathematical model of a typical Monopulse tracking seeker is presented. A three-degrees-of-freedom missile-target engagement simulation model is carried out under MATLAB/SIMULINK environment for testing and evaluation. Simulation results are given. The paper is terminated with conclusions.

\section{Keywords:}

Missile Radio Seeker, Homing Guidance Systems, ECM, ECCM 


\section{Introduction:}

The confrontation between radar designers and countermeasures designers has flourished rapidly and has become an extremely important motivation for new radar designs known as Electronic Counter Countermeasures (ECCM) as well as Countermeasures system design. ECM has been used as a force multiplier against defense network which has the problem of detecting and tracking the attackers through their jamming effects [1-11]. This problem can be diluted if the operators of the defense network utilize the provided electronic counter countermeasures capabilities in their equipment.

Different technical and tactical means are now available for jamming of semi-active radar homing guided missiles in order to maximize the enemy vehicle survival probability (minimize the kill probability by of missiles). However, the underlying missile system utilizes velocity tracking so that velocity deception ECM techniques are able to degrade its performance. Angle jamming of monopulse radar is difficult for the ECM designer because they are conceptually immune to amplitude-modulated ECM. However, there are different ECM techniques that have a degree of jamming effectiveness [4] [8].

The antenna of the single target-tracking radar will shift its tracking direction as the jammers are turned on and off provided that the noise jamming is of sufficient strength. When this technique is used against a homing missile in the Homing on Jam (HOJ) mode, the missile can be deceived and will either pass through the centroid of the multi target formation or veer off for a complete miss [7-9]. This means that the performance of the missile is seriously degraded and in most cases the missile mission is not fulfilled.

The paper starts by describing a model for a continuous wave (CW) seeker. The problem statement associated with the proposed ECCM circuit is highlighted. The proposed ECCM circuit contains mainly two circuits. The first one is the angle gate circuit and the other is angle offset circuit. The angle gate circuit is implemented for zeroing the angle tracker output whenever the measured angle error exceeds a certain threshold level. The angle offset circuit is implemented to improve the missile sensitivity to any abrupt change in the tracking error signal developed in the Monopulse receiver. This circuit also develops an equal and opposite error signal in order to switch an antenna to the opposite direction. Thus, the induced angular error will not be developed due to jamming and the antenna position will keep changing around the target LOS. A three-degrees-of-freedom missile navigation, guidance, and control model is carried out under MATLAB/SIMULINK environment. The seeker model associated with proposed circuitry of ECCM is integrated. Extensive simulation has been conducted and results are recorded for performance analysis. Finally, paper terminates with conclusion.

\section{Mono pulse tracking seeker Performance against Interference Signal:}

A semi-active homing system is one that selects and chases a target by following the energy from an external source, such as tracking radar, reflecting from the target. This illuminating radar may be ground-based, ship-borne, or airborne as shown in Fig. 1.

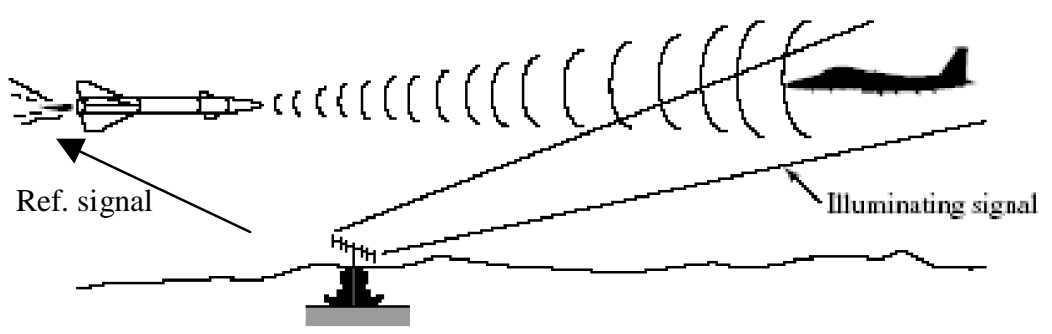

Fig. (1) Semi-active homing system

Semi-active homing requires the target to be continuously illuminated by the external radar at all times during the flight of the missile. The illuminating energy may be supplied by the target-tracking 
radar itself or by a separate transmitter collimated with it. The radar energy reflected by the target is picked up by a tracking receiver (radio seeker) in the nose of the missile and is used by the missile's guidance system.

The block diagram of a CW Doppler Monopulse tracking radar with additional proposed ECCM circuit is shown in Fig. 2. The Phase Lock Lope (PLL) is initially in lock without static phase error. i.e. the central angular frequency of the VCO is equal to the angular frequency of the useful input signal. The VCO output signal can be obtained as follows;

$$
y_{5}=a_{m} \cos \left(\omega_{I F 1}+\phi_{1}\right)
$$

where $\omega_{I F 1} / 2 \pi$ is the first IF carrier frequency of the useful signal and $\phi_{1}$ is the useful signal phase constant. The PLL contains a low path filter (LPF) whose transfer function is given by:

$$
F(S)=\left(1+\tau_{1} S\right) / \tau_{2} S
$$

Where $\tau_{1}$, and $\tau_{2}$ are the LPF time constants. The acquisition time $\left(T_{a c q}\right)$ is given by:

$$
T_{a c q}=\left(\omega_{I F 1}-\omega_{o}\right)^{2} /\left(2 \zeta \omega_{n}^{3}\right)
$$

where $\omega_{o} / 2 \pi$ is the VCO central frequency, $\omega_{o}$ is the loop natural frequency, and $\xi$, is the PLL damping ratio. In the presence of an unwanted signal, there are two sinusoids of different amplitudes and frequencies existing at the input of the PLL (y4). This happens in case of presence of interference signal or two targets in the FOV of the missile's front antenna. The sum channel signal at the output of the first mixer (y1 in Fig. 2) is given by [9], [11]:

$$
\begin{aligned}
y_{1} & =a_{1} \sin \left(\omega_{I F 1}+\phi_{1}\right)+a_{2}(t) \sin \left(\omega_{I F 1}+\phi_{2}\right) \\
& =a_{1}(t) \sin \left(\omega_{I F 1} t+\phi_{1}\right)+a_{2}(t) \sin \left(\omega_{I F 1} t+\phi_{1}+\Delta_{\omega} t\right) \\
& \left.=A(t) \sin \left[\omega_{I F 1} t+\phi_{1}+\delta(t)\right]\right)
\end{aligned}
$$

where $a_{1}$ is the amplitude of the original signal, $a_{2}$ is the amplitude of the interfering signal, $\omega_{I F 1} / 2 \pi$ is the first IF frequency of the useful signal in (hertz), and $\Delta_{\omega}$ is the frequency difference between the useful and interference signal (hertz). In addition, $A(t)$ and $\delta(t)$ are the time varying amplitude and time varying phase of the input signal to the PLL respectively, and are given by:

$$
\begin{aligned}
& \delta(t)=\tan ^{-1} \frac{R \sin \left(\Delta_{\omega} t\right)}{1+R \cos \left(\Delta_{\omega} t\right)} \\
& A(t)=a_{1} \sqrt{1+R^{2}+2 R \cos \left(\Delta_{\omega} t\right)}
\end{aligned}
$$

Where $\mathrm{R}=\mathrm{a} 2 / \mathrm{a} 1$ is the amplitude ratio of the two input signals. It is noted that amplitudes and phases have fundamental frequency of $\Delta_{\omega}$. The mixers outputs of the difference channels $y_{1}$ and $y_{2}$ are given, respectively as follows [9]:

$$
\begin{aligned}
& y_{2}=\left[a_{1} d_{p 1} \sin \left(\omega_{I F 1} t+\phi_{1}\right)+a_{2} d_{p 2} \sin \left(\omega_{I F} t+\phi_{1}+\Delta_{\omega} t\right] \cdot \sin \left(\omega_{1} t\right)\right. \\
& y_{3}=\left[a_{1} d_{y 1} \sin \left(\omega_{I F 1} t+\phi_{1}\right)+a_{2} d_{y 2} \sin \left(\omega_{I F 1} t+\phi_{1}+\Delta_{\omega} t\right] \cdot \cos \left(\omega_{1} t\right)\right.
\end{aligned}
$$

Where ${ }^{d_{p 1}}$ and dp2 are proportional to the angles between the LOS of the two targets and the off bore sight axis of the antenna in pitch plane. Also $d_{y 1}$ and $d_{y 2}$ are proportional to the angles between the LOS of the two targets and the off bore sight axis of the antenna in yaw plane. 
The sum and difference signals $y_{1}, y_{1}$ and $y_{3}$ are amplified by the AGC amplifier the output of the AGC amplifier is given by:

$$
\begin{gathered}
y_{4}=\frac{\left[v_{o} d_{p 1} \sin \left(\omega_{I F 1} t+\phi_{1}\right)+v_{o} d_{p 1} R \sin \left(\omega_{I F 1} t+\phi_{1}+\Delta_{\omega} t\right)\right] \sin \left(\omega_{L} t\right)}{\sqrt{1+R^{2}+2 R \cos \left(\Delta_{\omega} t\right)}} \\
+\frac{\left[v_{o} d_{y 1} \sin \left(\omega_{I F 1} t+\phi_{1}\right)+v_{o} d_{y 2} R \sin \left(\omega_{I F 1} t+\phi_{1}+\Delta_{\omega} t\right)\right] \cos \left(\omega_{L} t\right)}{\sqrt{1+R^{2}+2 R \cos \left(\Delta_{\omega} t\right)}} \\
+\frac{\left[v_{o} \sin \left(\omega_{I F 1} t+\phi_{1}\right)+v_{o} R \sin \left(\omega_{I F 1} t+\phi_{1}+\Delta_{\omega} t\right)\right]}{\sqrt{1+R^{2}+2 R \cos \left(\Delta_{\omega} t\right)}}
\end{gathered}
$$

For wide band AGC, the AGC amplifier has a gain $\mathrm{G}$ that can be defined as follows:

$$
\mathrm{G}=\mathrm{Vo} / \mathrm{A}(\mathrm{t})
$$

Where $V_{o}$ is the signal amplitude at the AGC output. A parasitic dc component seems to appear at the phase detector output, which is canceled by the VCO via phase shift. At this time the VCO output signal (y5) is given by:

$$
y_{5}=a_{m} \cos \left[\omega_{I F 1} t+m \sin \left(\Delta_{\omega} t-\alpha\right)\right]=a_{m} \cos \left(\omega_{I F 1} t+\psi\right)
$$

Where $\psi=m \sin \left(\Delta_{\omega} t-\alpha\right)$ stands for the parasitic frequency modulation of the VCO, with $\mathrm{m}$ and $\alpha$ being arbitrary constants, the term $\omega_{I F 1}$ means that the VCO is supposed to remain synchronous with the input useful signal, i.e. the PLL remain lock on the useful signal. This output is mixed with the AGC amplifier output yielding an error used to drive the VCO as:

$$
y_{7}=y_{4} \cdot y_{5}
$$

The output signal of the $\operatorname{VCO}\left(y_{5}\right)$ is phase shifted by $90^{\circ}$ so that the input to the phase detector PD2 is:

$$
y_{6}=a_{m} \sin \left(\omega_{I F 1} t+\psi\right)
$$

This phase detector carries on a coherent detection for the output of the AGC Amplifier y4 yielding the output ${ }^{y_{8}}$, which is filtered to yield the signal ${ }^{y_{9}}$ as follows:

$$
\begin{aligned}
y_{9}= & y_{4} \cdot y_{5} \\
= & \left(\frac{a_{m} v_{o}}{2}\right) \frac{\left[d_{p 1}+d_{p 2} R \cos \left(\Delta_{\omega} t\right)\right.}{\sqrt{1+R^{2}+2 R \cos \left(\Delta_{\omega} t\right)}} \cdot \sin \omega_{1} t \\
& +\left(\frac{a_{m} v_{o}}{2}\right) \frac{\left[d_{y 1}+d_{y 2} R \cos \left(\Delta_{\omega} t\right)\right.}{\sqrt{1+R^{2}+2 R \cos \left(\Delta_{\omega} t\right)}} \cdot \cos \omega_{1} t
\end{aligned}
$$

The control signal to the antenna seeker is then recovered by resolving y9 to its Cartesian components by multiplying it by $\sin \omega_{1}$ and $\cos \omega_{1} \mathrm{t}$ and filtering out the high frequency terms as follows:

$$
\begin{aligned}
& y_{10}=\frac{a_{m} v_{o}}{4}\left[A d_{p 1}+B d_{p 2}\right] \\
& y_{11}=\frac{a_{m} v_{o}}{4}\left[A d_{y 1}+B d_{y 2}\right]
\end{aligned}
$$


$y_{10} \& y_{11}$ represent two output errors of the monopulse tracking seeker. These errors are applied to a proposed ECCM circuit, which adjusted to a certain threshold voltages in order to control the antenna main beam width and makes the monopulse tracking seeker tracks the attacking target properly in spite of presence of jamming on it.

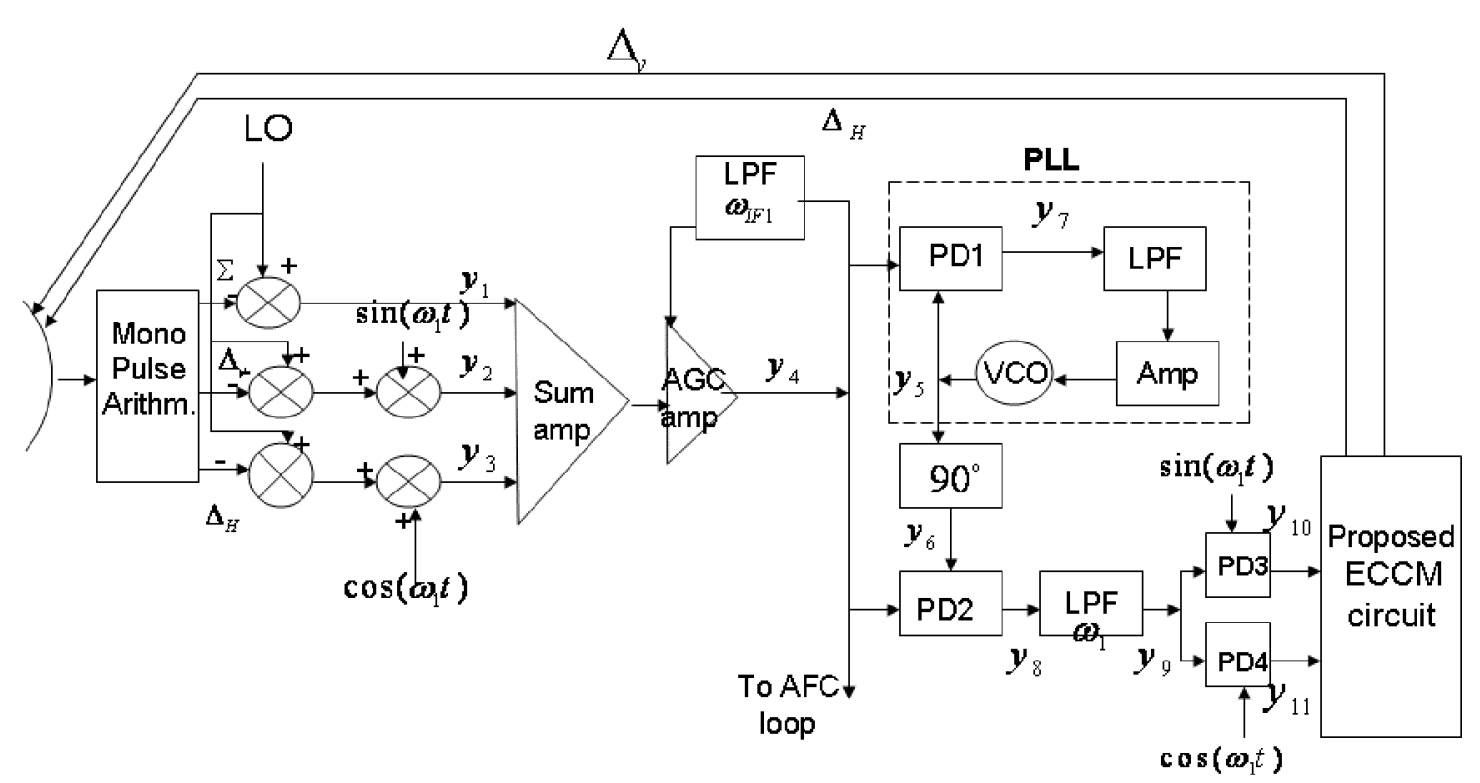

Fig. 2 Block diagram of monopulse tracking radio seeker with proposed ECCM circuit

\section{Proposed ECCM Circuit Description}

Angle deception ECM techniques are considered the most effective jamming techniques facing the semi-active radio guided missiles. In order to overcome their effects, the missile must apply more than one ECCM technique simultaneously. Thus, this paper proposes an ECCM in addition to its logic circuit implementation against angle deception techniques. Then it demonstrates its effect on a missile system under static and dynamic missile flight conditions in the presence of angle deception ECM. The proposed ECCM is a hybrid technique applicable to CW Doppler monopulse seekers, where it is based on the following ECCM techniques:

\subsection{Angle gate}

This technique is implemented by using a control circuit for zeroing the angle tracker output whenever the measured angle error exceeds a certain threshold level. This circuit is not used during the initial target acquisition. By using this technique the effective missile antenna beam width is reduced to approximately the set reference angle, providing fine angle discrimination. In addition, it decreases the effect of multiple target barrages jamming, multiple target blinking jamming, side lobe standoff jamming, and some forms of main lobe standoff jamming [9-11].

\subsection{Angle Offset}

This technique is implemented to improve the missile sensitivity to any abrupt change in the tracking error signal developed in the mono pulse receiver. In addition, it develops an equal and opposite error signal in order to switch the antenna to the opposite direction. Thus, the induced angular error will not be developed due to jamming and the antenna position will keep changing around the target LOS. 


\subsection{Servo-Bandwidth Variation}

This technique is implemented by controlling the servo-bandwidth as a function of time such that the longer the target-missile range, the less servo bandwidth is required. That is, the servo-bandwidth is controlled to be initially small and gradually increases with time. This small initial value of bandwidth reduces angle sensitivity and slows down the response, in order to reduce susceptibility to on-target angle-deception ECM techniques [11].

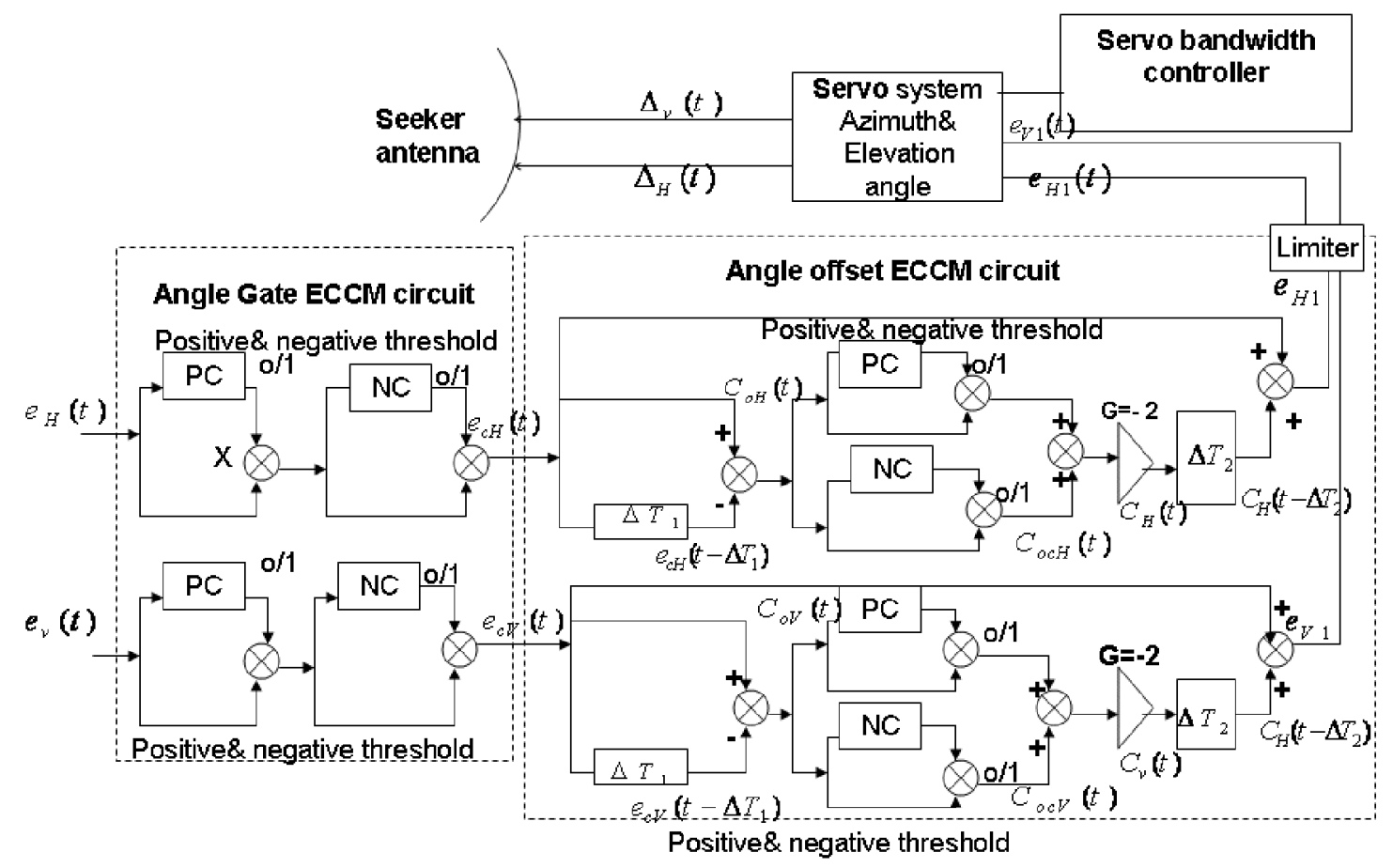

Fig. 3 functional block diagram of the proposed ECCM circuit

The output of the angle-gate circuit in each channel is given as follows:

$$
\begin{aligned}
& e_{c V}(t)= \begin{cases}e_{V}(t) & \text { If }\left|\mathrm{e}_{\mathrm{V}}(t)\right| \leq \mid \text { threshold value } \mid \\
0 & \text { If }\left|\mathrm{e}_{\mathrm{V}}(t)\right| \mathrm{f} \mid \text { threshold value } \mid\end{cases} \\
& e_{c H}(t)= \begin{cases}e_{H}(t) & \text { If }\left|\mathrm{e}_{\mathrm{H}}(t)\right| \leq \mid \text { threshold value } \mid \\
0 & \text { If }\left|\mathrm{e}_{\mathrm{H}}(t)\right| \mathrm{f} \mid \text { threshold value } \mid\end{cases}
\end{aligned}
$$

Then these signals are applied to the angle-offset circuit. Within the angle-offset circuit, the input signal is split into three signals: one of them is delayed by $\Delta T_{1}$ yielding $e_{c}\left(t-\Delta T_{1}\right)$, and then compared with the input signal ec(t) (second) via a comparator. If there is any abrupt change in the input tracking error signal ec(t) due to jamming effect, it will be sensed by the comparator yielding an output with amplitude which is related to that abrupt change value, and is given by:

$$
\begin{aligned}
& C_{o H}(t)=e_{c H}(t)-e_{c H}\left(t-\Delta T_{1}\right) \\
& C_{o V}(t)=e_{c V}(t)-e_{c V}\left(t-\Delta T_{1}\right)
\end{aligned}
$$

In order to reset the effect of the angle-offset circuit whenever there is no abrupt change in the input tracking error, the comparator output is controlled to be "0" whenever it does not exceed a certain threshold value. Thus, the comparator output $\mathrm{Co}(\mathrm{t})$ is applied to two parallel controllers, which are adjusted at positive and 
negative thresholds. The threshold values are kept small enough in order to detect a very small abrupt change in the tracking error. At this time the two parallel controllers output will be equal to the abrupt change value, and is given by:

$$
\begin{aligned}
& e_{o c H}(t)= \begin{cases}C_{o H}(t) & \text { If }\left|\mathrm{C}_{\mathrm{oH}}(t)\right| \geq \mid \text { threshold value } \mid \\
0 & \text { If }\left|\mathrm{C}_{\mathrm{oH}}(t)\right| \mathrm{p} \mid \text { threshold value } \mid\end{cases} \\
& e_{o c V}(t)= \begin{cases}C_{o V}(t) & \text { If }\left|\mathrm{C}_{\mathrm{ov}}(t)\right| \geq \mid \text { threshold value } \mid \\
0 & \text { If }\left|\mathrm{C}_{\mathrm{oV}}(t)\right| \mathrm{p} \mid \text { threshold value } \mid\end{cases}
\end{aligned}
$$

Then this signal is amplified with gain $(\mathrm{G}=-2)$ to develop a control signal, which is used in the offset operation, and is given by:

$$
\begin{aligned}
& C_{H}(t)=-2 C_{o c H}(t) \\
& C_{V}(t)=-2 C_{o c V}(t)
\end{aligned}
$$

This signal is delayed again by $\Delta T_{2}$ and added to the output of the angle-gate circuit output $e_{c}$ (t) to carry out the offset operation. The resulting signal in each channel is given as follows:

$$
\begin{aligned}
& e_{H 1}(t)=e_{c H}(t)+C_{H}\left(t-\Delta T_{2}\right) \\
& e_{V 1}(t)=e_{c V}(t)+C_{V}\left(t-\Delta T_{2}\right)
\end{aligned}
$$

The signal $e_{1}(t)$ is then applied to a limiter in order to limit any unwanted overshooting in the circuit reactions. The upper and lower threshold values of this limiter are chosen to be related to the main antenna beam width in order to keep the signal in continuous processing used in the offset operation, and is given by:

$$
\begin{aligned}
& C_{H}(t)=-2 C_{o c H}(t) \\
& C_{V}(t)=-2 C_{o c V}(t)
\end{aligned}
$$

This signal is delayed again by $\Delta T_{2}$ and added to the output of the angle-gate circuit output $e_{c}$ (t) to carry out the offset operation. The resulting signal in each channel is given as follows:

$$
\begin{aligned}
& e_{H 1}(t)=e_{c H}(t)+C_{H}\left(t-\Delta T_{2}\right) \\
& e_{V 1}(t)=e_{c V}(t)+C_{V}\left(t-\Delta T_{2}\right)
\end{aligned}
$$

The signal $e_{1}(t)$ is then applied to a limiter in order to limit any unwanted overshooting in the circuit reactions. The upper and lower threshold values of this limiter are chosen to be related to the main antenna beam width in order to keep the signal in continuous processing.

\section{Static-Circuit Response to Tracking Error Signals}

The proposed ECCM circuit is modeled using the SIMULINK as shown in Fig. 4, where it consists of angle-gate and angle-offset circuits. These circuits are connected in series with the servo bandwidth controller. The input of this model is the output tracking error signal obtained from the mono pulse tracking system. The model output is applied to the antenna servo transfer function which is given as follows [9]:

$$
\frac{\theta_{a n t}}{\lambda}=\frac{3556 s^{2}+1095248 s+38120320}{s^{4}+1372 s^{3}+123743 s^{2}+4849953 s+38120320}
$$

The two serial controller devices of the angle-gate circuit are simulated by two relays, which are adjusted by positive and negative thresholds. In this model the two positive and negative thresholds 
error voltages are adjusted at 0.8 and -0.8 volt respectively, in order to control the antenna main beam width by gate width equals 4 degrees, in addition, the two parallel controller of the angle-offset circuit are simulated by two relays, which are adjusted at 0.6 and -0.6 volt. These threshold values are chosen to pass the tracking error signal of the tracked target that produces a high maneuver without any distortion.

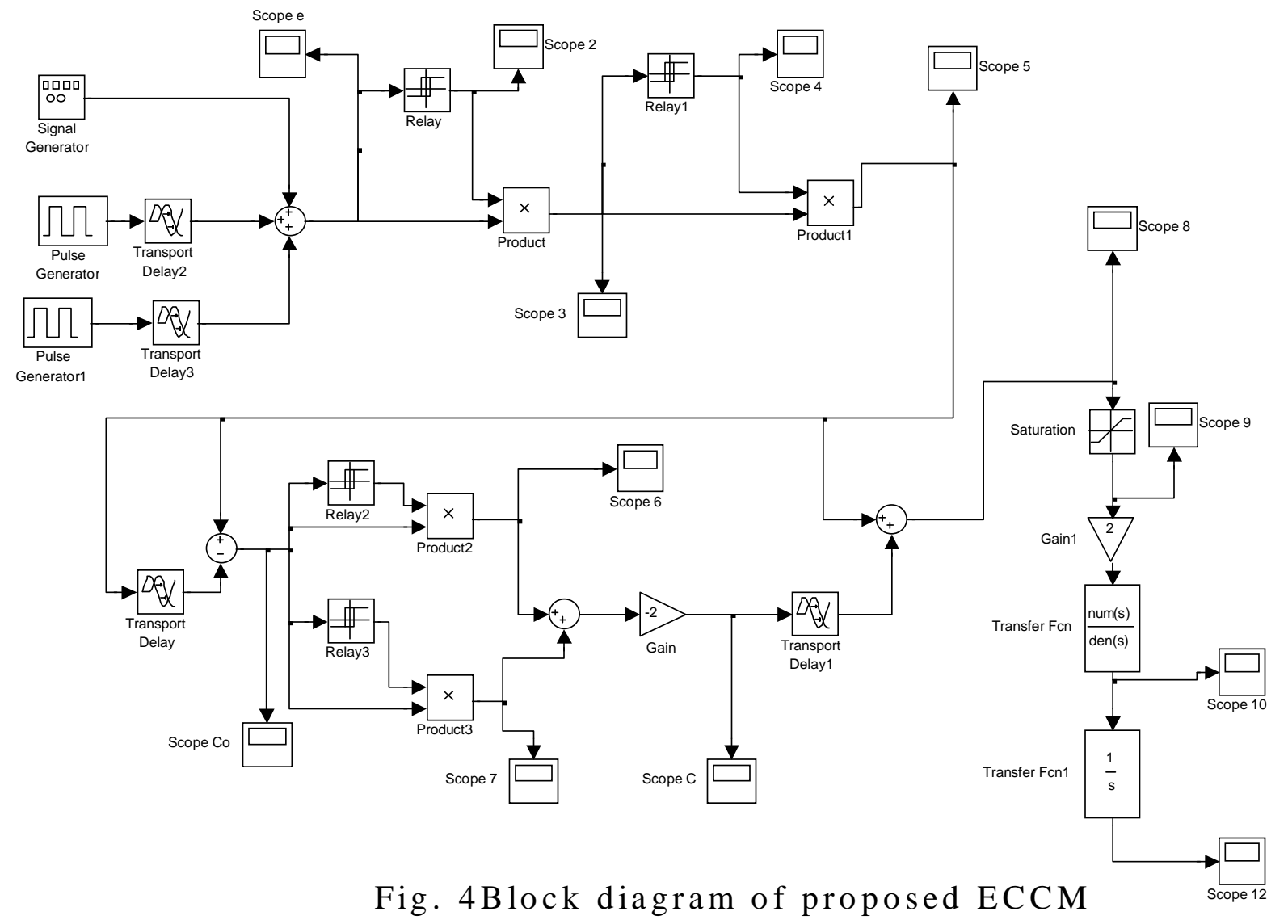

\section{Case 1: Response without jamming}

The simulated tracking error signal (e) is shown in Fig. 5. During the operation of the angle-gate circuit, the tracking error signal (e) is within the angle gate width. Thus, the (e) signal passes the anglegate circuit without any distortions, and then it is applied to the angle-offset circuit. Within this circuit the comparator does not detect any abrupt change in the (e) signal. Thus, the comparator output (Co) shown in Fig.6, is neglected by the two parallel controller devices, and the final addition of offset signal (C) is zero, Fig. 7. 


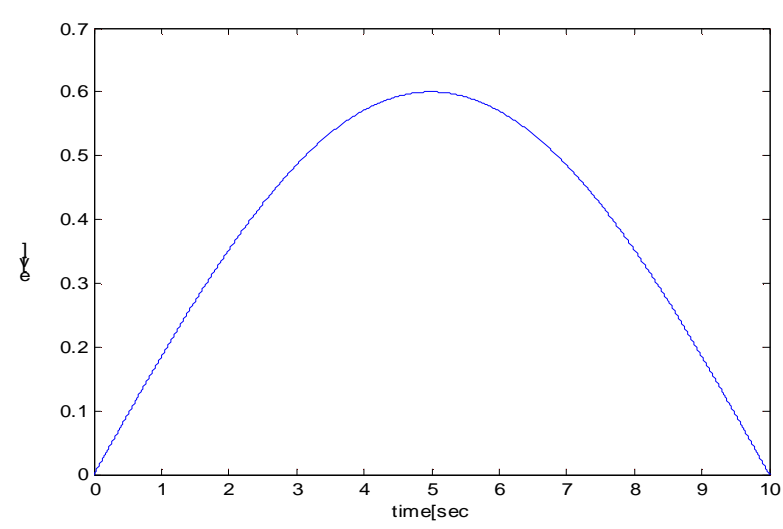

Fig.5. Tracking error signal $\mathrm{e}(\mathrm{t})$

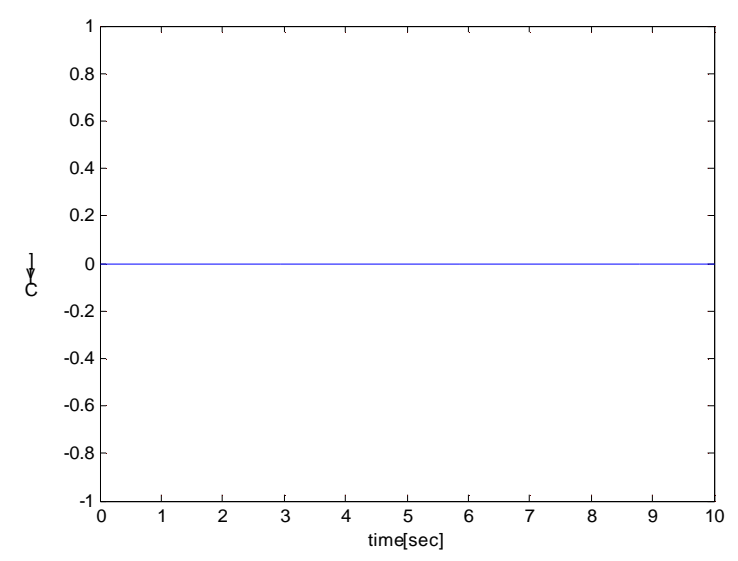

Fig.7. Additional Offset Signal

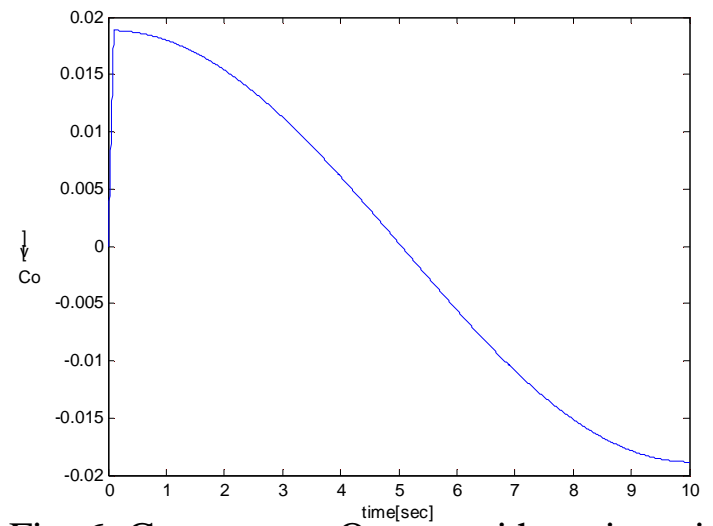

Fig. 6. Comparator Output without jamming

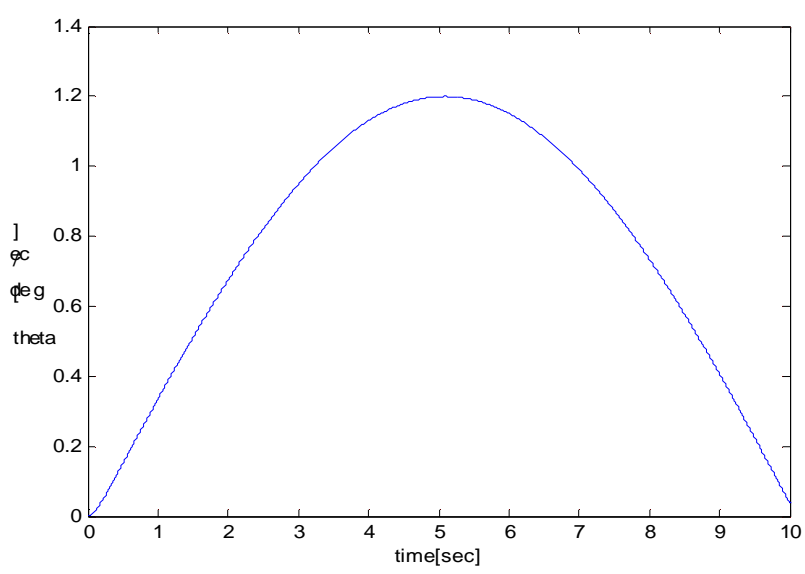

Fig.8. Antenna heading angle

\section{Case 2: Response with blinking jamming}

Assume that the tracked target is centered between two blinking jammers, which are located at angular positions $5^{\circ}$ and $-5^{\circ}$ w.r.t. the tracked target LOS. Thus, a blinking signal with $25 \%$ duty cycle is produced. Applying the proposed ECCM circuit, the jamming effectiveness is degraded, and the antenna heading keeps tracking on the target LOS with very small angular error. The input tracking error signal to the model circuit is shown in Fig. 9, where the blinking jamming starts at $t=5$ sec. Within the angle-gate circuit, the first controller produces a control signals (0/1), Fig. 10, for zeroing the induced angular error by the first jammer, Fig.11. While the second controller produces a control signal as shown in Fig.12, for zeroing the induced angular error by the second jammer, Fig. 13. Then, the circuit output signal $(\mathrm{x} 4)$ is applied to the angle-offset circuit. 


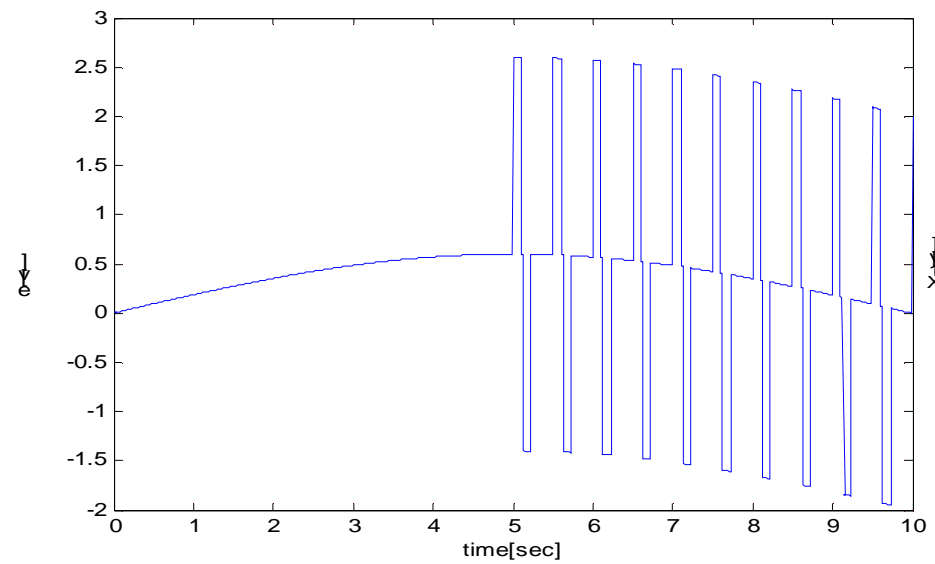

Fig.9 Tracking Error signal with blinking jamming effect

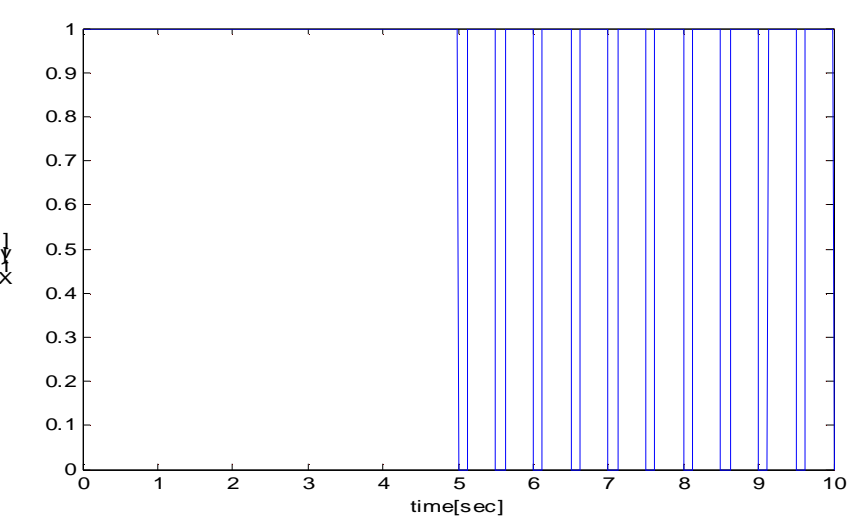

Fig. 10 First Controller output signal

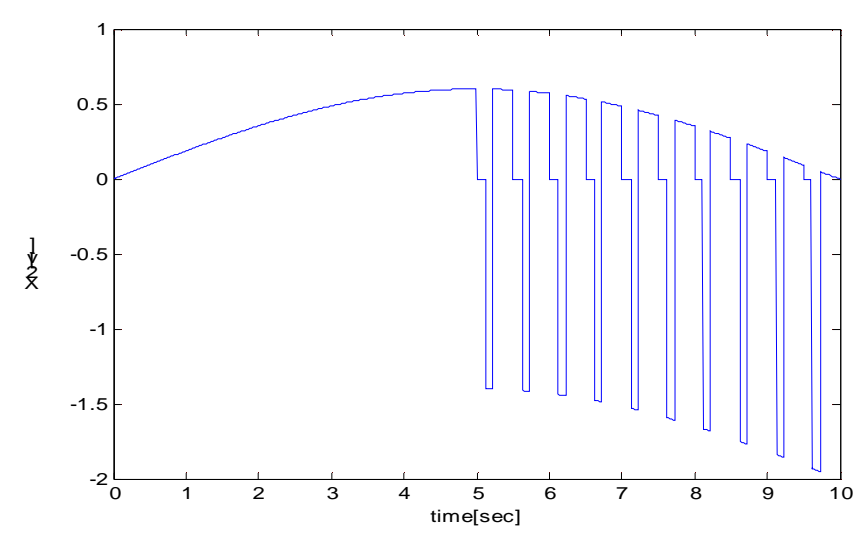

Fig. 11 Tracking Error signal with first Controller

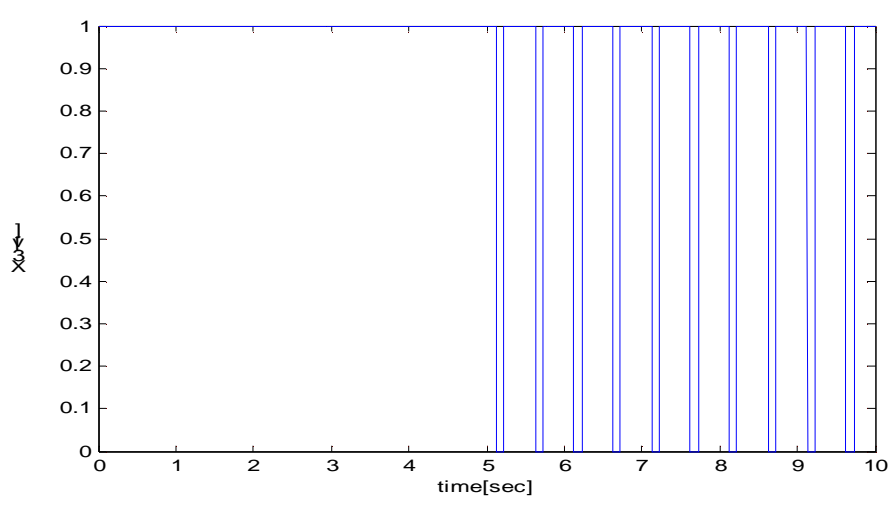

Fig. 12 Second Controllers output Signal effect

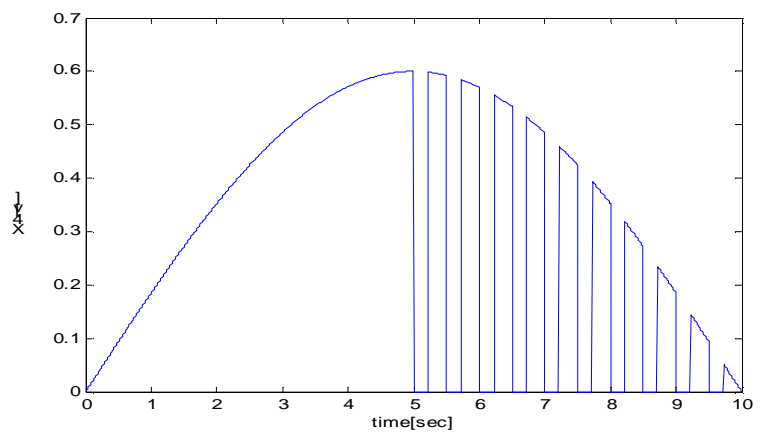

Fig. 13 Tracking Error signal with null effect

Within the angle-offset circuit, the abrupt change in the input signal ( $x 4)$ is sensed and offset signals corresponding to this change are developed, which are equal in amplitude and opposite in direction w.r.t that change. The comparator output signal (Co) presents the sensed abrupt change in its input signal, Fig.14, while the two parallel controller devices work for zeroing the Co signal whenever it does not 
exceed the adjusted thresholds. Then the resulting signal is amplified by gain $(\mathrm{G}=-2)$ in order to produce the additional offset signal, Fig. 15, which is added to the angle gate circuit output (x4) in order to produce the offset angular error in the opposite direction, Fig. 16. The resulting antenna heading keeps tracking on the target LOS with small angular error, Fig. 17. The antenna LOS angle, with and without applying the MBJ ECM technique is shown in Fig.18, since it is obtained by integrating the heading angle with time. It is clear that at the time of starting the blinking jamming the antenna will be deviated away from the target LOS, and then starts oscillating between the two jammers angular positions. However, the target LOS will deviates about one degree, but target will stay in the FOV missile seeker antenna. The antenna succeeds to hack on the target LOS.

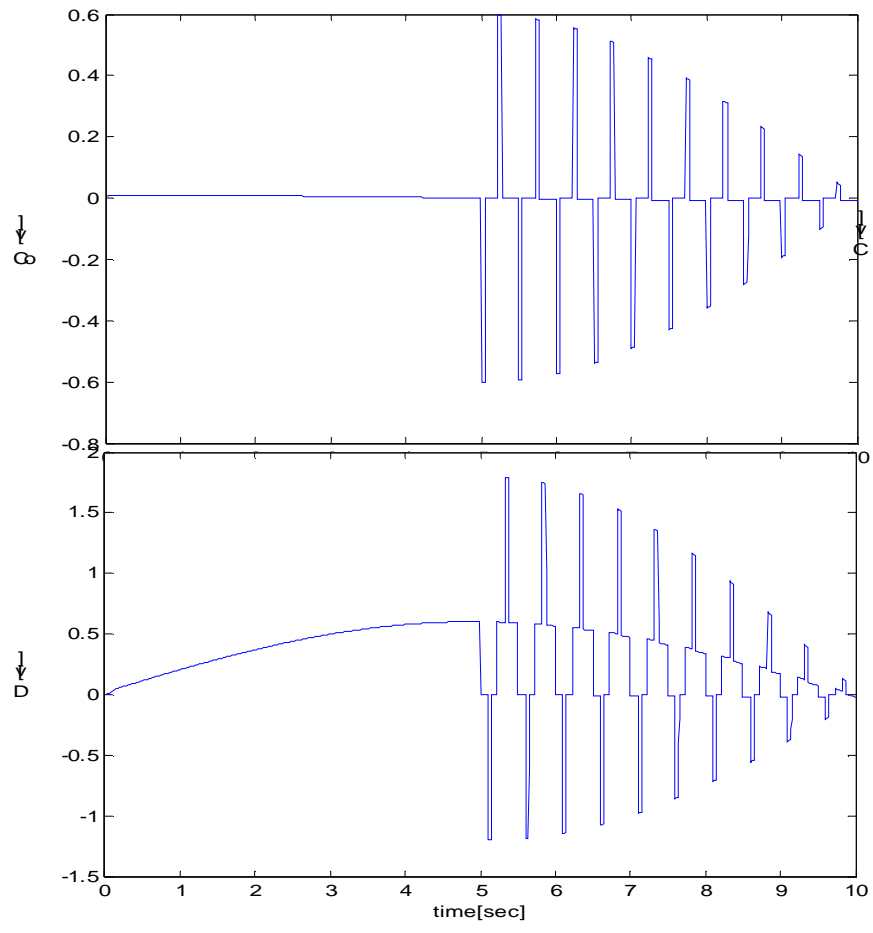

Fig. 16 Input Tracking Error to the antenna servo after processing

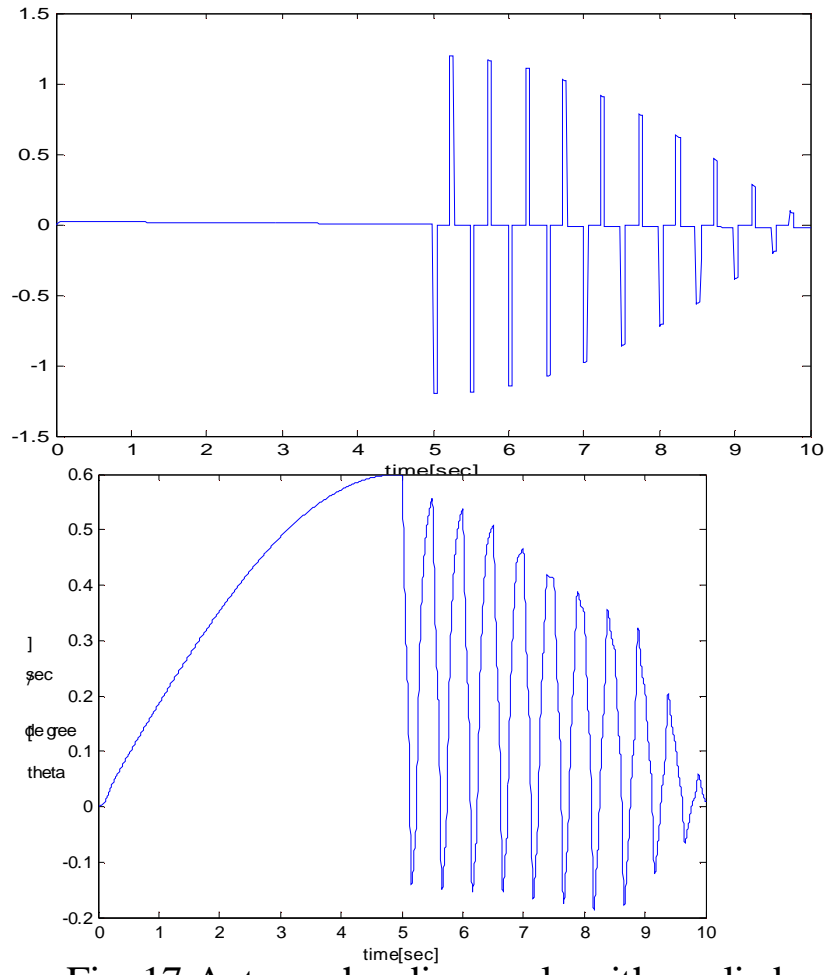

Fig. 17 Antenna heading angle with applied ECCM

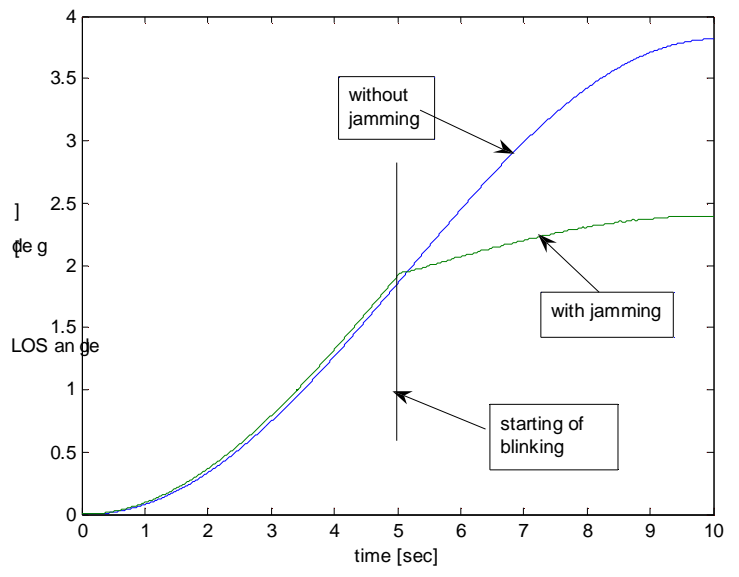

Fig. 18 LOS angle with and without applied jamming 


\section{Case 3: Response with Abrupt Change in the Tracking Error}

Due to jamming an abrupt change can occur in the tracking error. This change can be sensed by the angle-offset circuit, which develops an equal and opposite error signal used to force the antenna into the opposite direction. This process may be monitored to ensure closed loop operation, and to prevent error accumulation, which might yield the antenna position to keep changing around the target LOS. The simulated input tracking error signal (e) to the proposed ECCM is shown in Fig. 19, which attains -0.6 volt abrupt change in its value at $\mathrm{t}=5 \mathrm{sec}$. However, the target LOS angle is still within the angle gate thresholds, which is adjusted to be -0.8 : 0.8 . Thus, the input error signal is able to pass the angle-gate circuit without any processing. Within the angle offset-circuit, the abrupt change ( 0.6 volt) in the input signal (x4) is sensed, and an offset signal corresponding to this change is developed, which is equal in amplitude and opposite in direction w.r.t that change. The comparator output signal (Co) presents the sensed abrupt change in its input signal, Fig. 20, while the two parallel controller devices work for zeroing the Co signal whenever it does not exceed the adjusted thresholds. Then the resulting signal is amplified by gain $(\mathrm{G}=-2)$ to produce the additional offset signal, Fig. 21, which is added to the angle-gate circuit output (x4) for producing the offset angular error in the opposite direction. The resulting antenna heading keeps tracking on the target LOS with small angular error, Fig. 22.

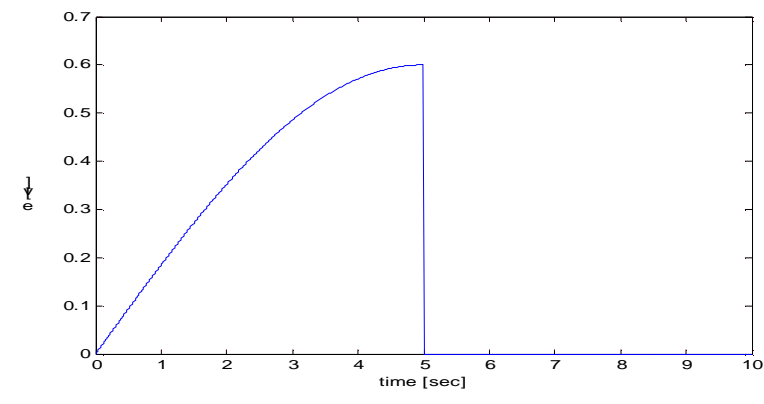

Fig. 19 Tracking error signal

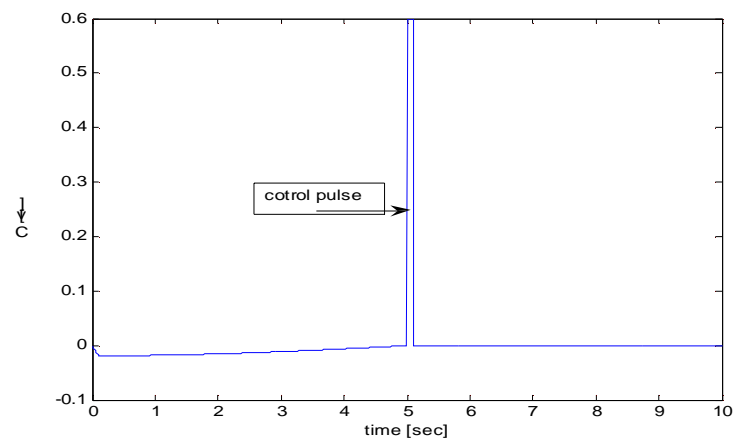

Fig. 21 Additional Offset Signal

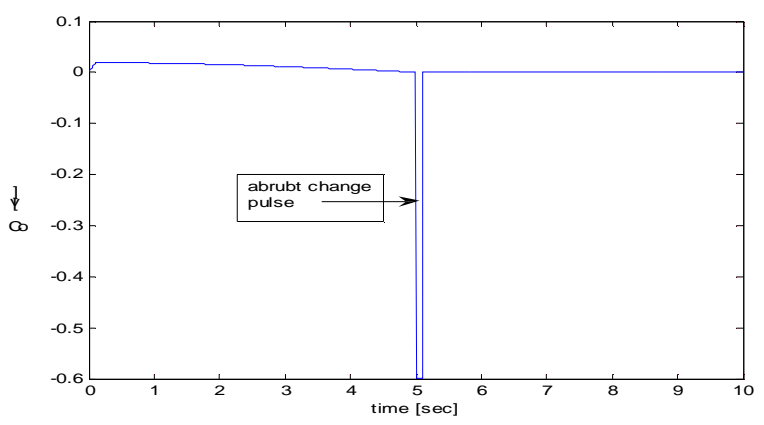

Fig. 20 Comparator Output

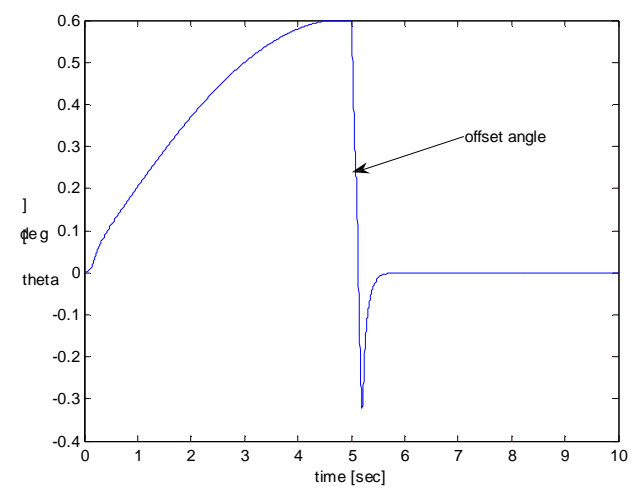

Fig. 22 Antenna heading angle

\section{Integrated Seeker with Missile Guidance and Control Model}

In this section, an integrated seeker model associated with the proposed ECCM circuit with missile guidance and control process is presented. The purpose of integration is to measure the performance of the proposed technique in typical missile target engagement scenarios. Random target maneuvers are other uncertain state for the radio seeker. Miss distance and missile dynamic are recorded for performance measure. Well-known proportional navigation [12-13] guidance method is utilized for 
steering the missile. Extensive simulation has been conducted according to target parameters in Table. 1.

Missile Response to MBJ:

The MBJ method attempts to attack the tracking dynamics of the angle-tracking radar. The simulated MBJ ECM scenario is shown in Fig. 23, where there are two blinking jammers staying behind the attacking target, and out of the missile coverage range. The jammer sources turn ON and OFF by rate within the pass band of the angle-tracking servo (e.g., 0.1 to $10 \mathrm{~Hz}$ ). In the shown scenario one of the jamming aircraft is designed to be a master and the other one is slave. The master aircraft provides master control of the sequential blinking by radiating its blinked noise into an antenna on the other slave aircraft. The noise power provided by the two jamming aircraft must be higher than that radiated by the attacking aircraft as their distances to the missile are greater. There is a time delay $\left(t_{s}\right)$ involved between the time that the master jammer signal is tuned OFF and the time that the slave noise jammer is turned ON.

Table-1 Simulated Targets Parameters

\begin{tabular}{|l|l|l|}
\hline Target and $\begin{array}{c}\text { Jammers } \\
\text { Parameters }\end{array}$ & Attacking target initial range & $10 \mathrm{Km}$ \\
\cline { 2 - 3 } & Initial range of jamming targets & $20 \mathrm{Km}$ \\
\cline { 2 - 3 } & Distance between the two blinking jammer targets & $1 \mathrm{Km}$ \\
\cline { 2 - 3 } & Attacking target speed & $0.4 \mathrm{Km} / \mathrm{sec}$ \\
\cline { 2 - 3 } & Target maneuver & $0.0 \mathrm{deg} / \mathrm{sec}$. \\
\cline { 2 - 3 } & Jammers power & 1000 watt \\
\cline { 2 - 3 } & Bblinking Rate & $2 \mathrm{~Hz}$ \\
\hline
\end{tabular}

The integration step size is fixed for the most flight $(\Delta t=0.1 \mathrm{sec})$. The program is terminated when the closing velocity changes its sign, which means that the relative range between missile and target is the minimum. The simulation results are carried out for four runs. The first two are conducted to one jamming -BJ-with and without applying the proposed ECCM while results are presented in Fig. 24-27. The second two scenarios are conducted for MBJ with ECCM and without ECCM as shown in Figs. 2833. The missile missed the target in both cases when the ECCM circuits are not utilized. While with applying the proposed ECCM the missiles seeker is able in both cases to degrade the effectiveness of the $\mathrm{BJ}$ and MBJ. The missile satisfactorily hits the attacking target with small miss-distance (one meter in case $\mathrm{BJ}$ and 15 meters in case of MBJ).

While considering noisy data in the presence of various sources of uncertainties the following results are recorded in Table 2. The proposed ECCM circuit the missile will be able to degrade the effectiveness of a uniform noise yet severe target maneuvers in presence of noise and jamming is an environment for more research to be conducted. 


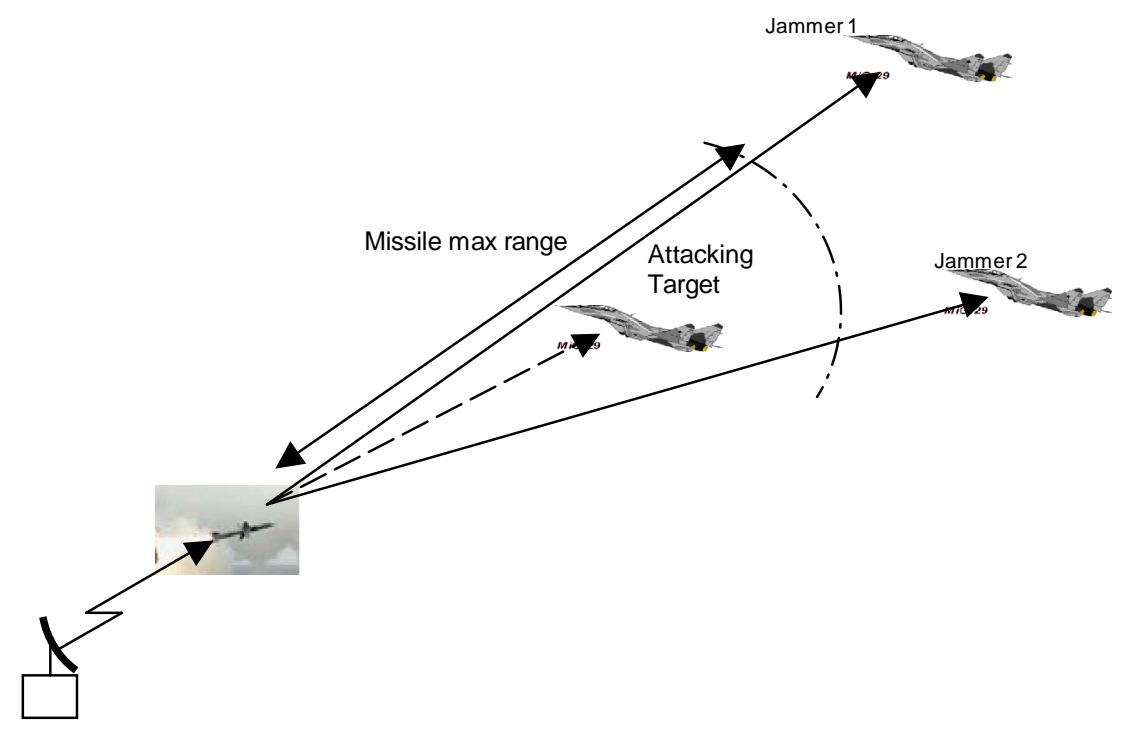

Fig. 23 Multiple blinking jammers ECM Scenario

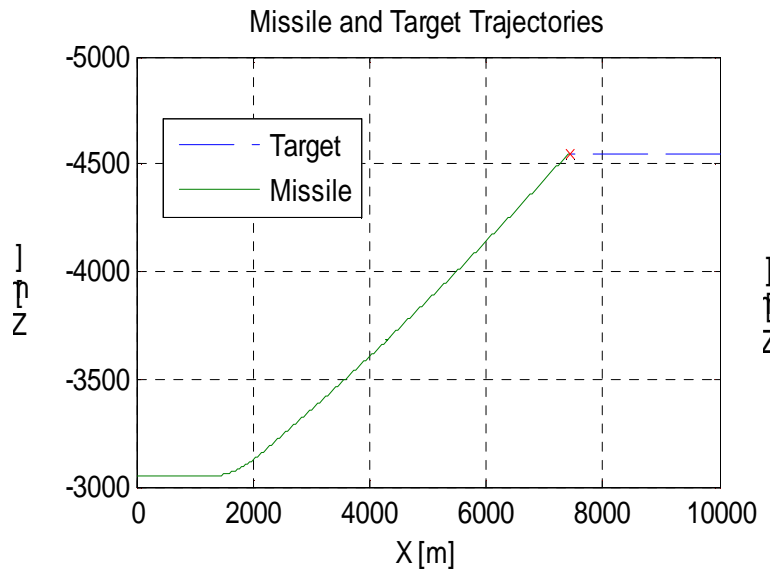

(a)

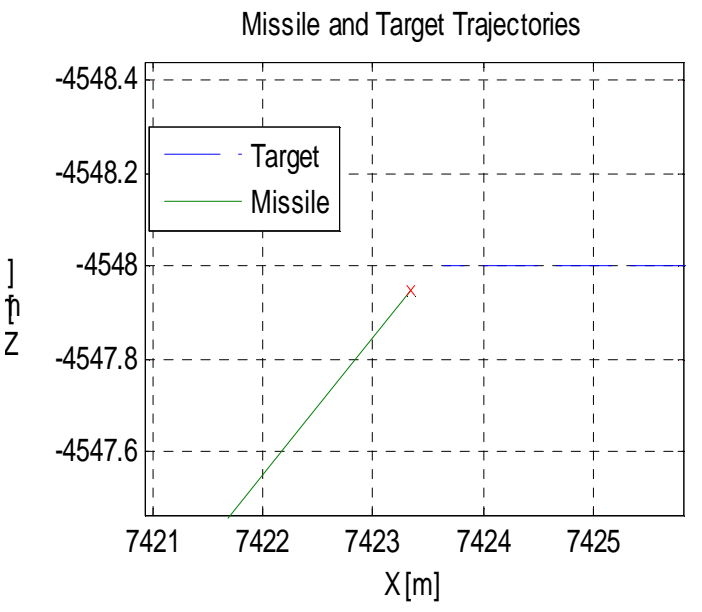

(b) focusing at impact point

Fig. 24 Missile Target Trajectory in presence of BJ with ECCM circuit

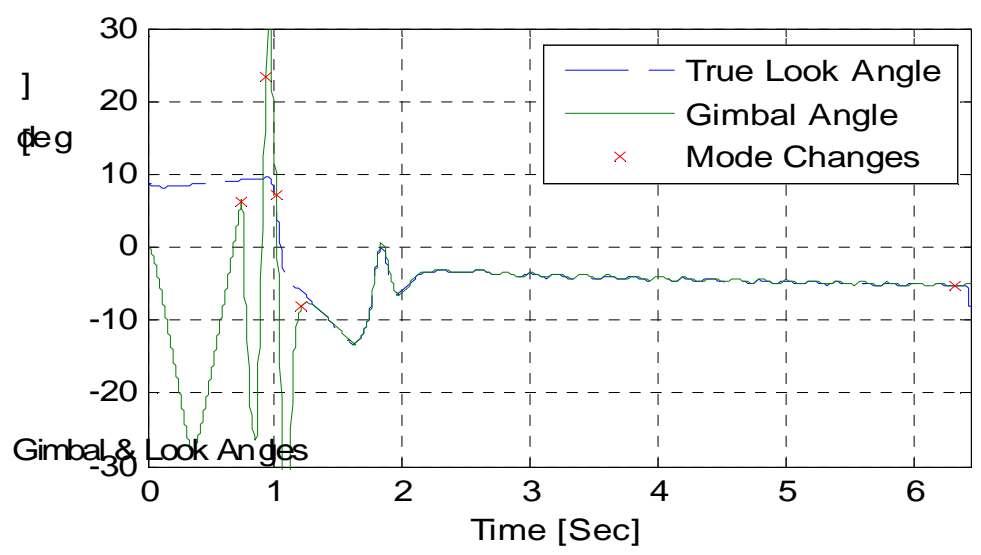


Fig. 25 Gimbal and look angle in presence of BJ with ECCM circuit

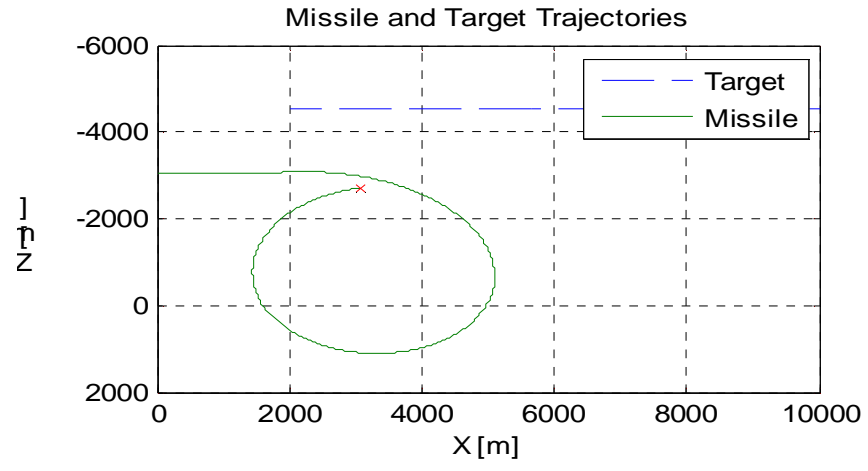

Fig. 26 Missile and Target Trajectory without ECCM circuit

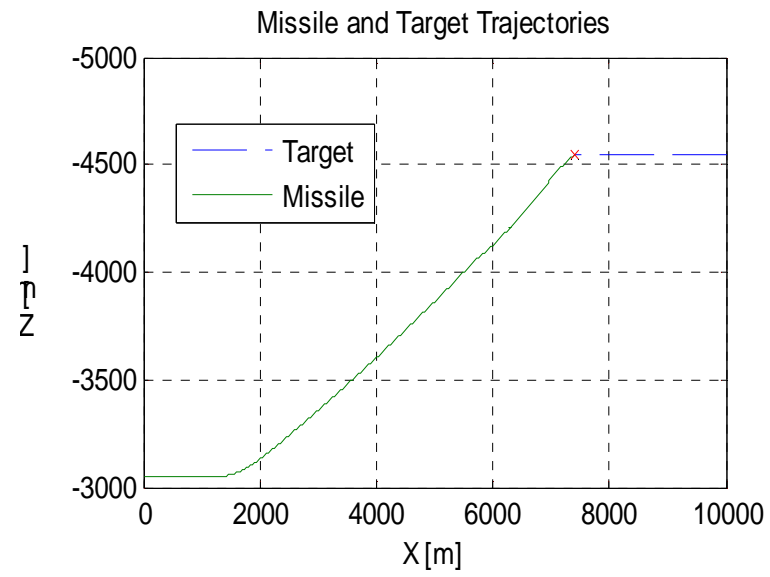

a)

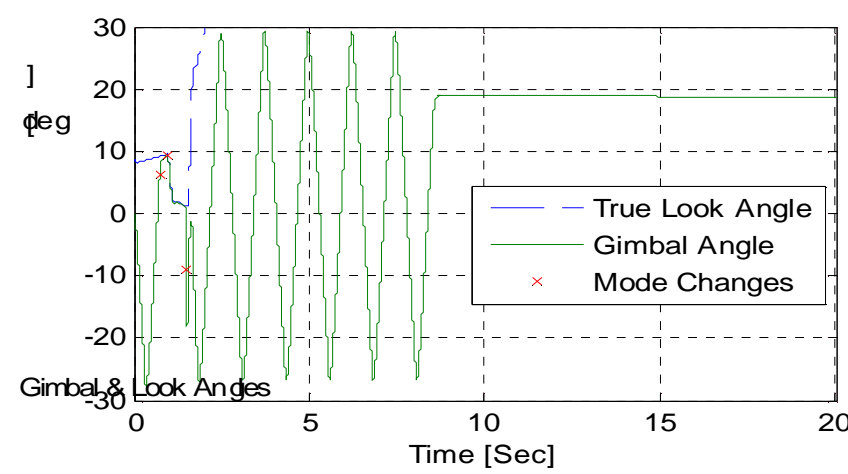

Fig. 27 Gimbal and Look angle without ECCM circuit

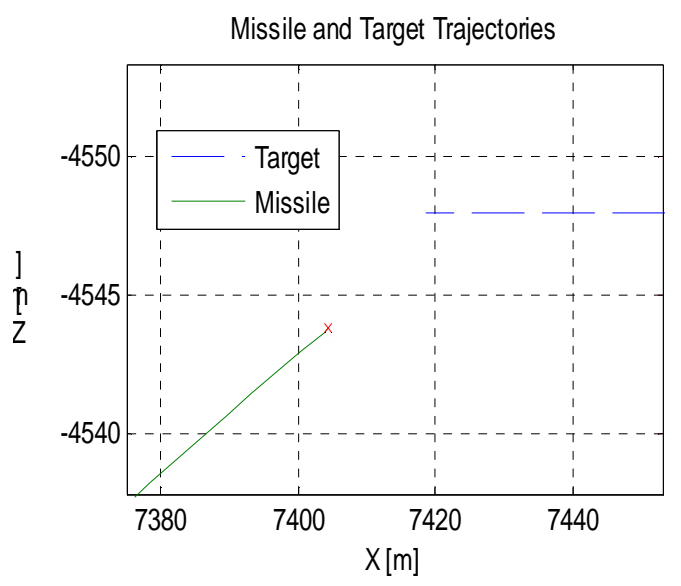

b) Focusing at impact point

Fig. 28 Missile and target trajectory in presence of MBL with ECCM circuit

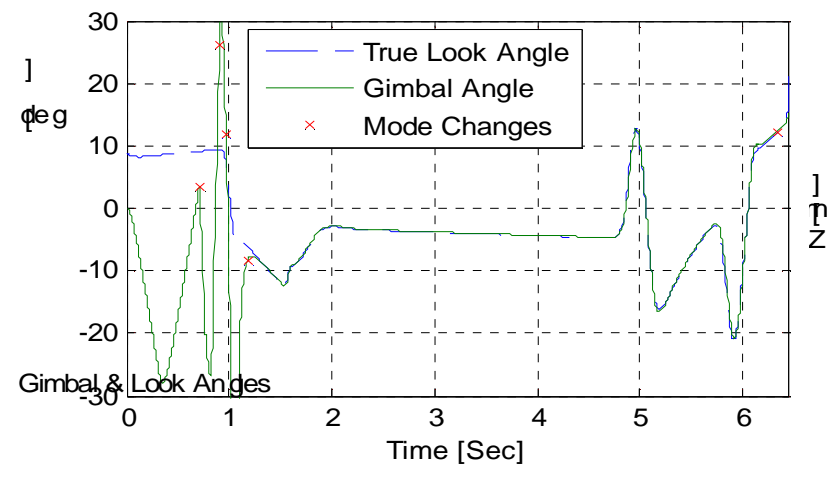

Fig. 29 Gimbal and look angle in presence of MBJ with ECCM circuit

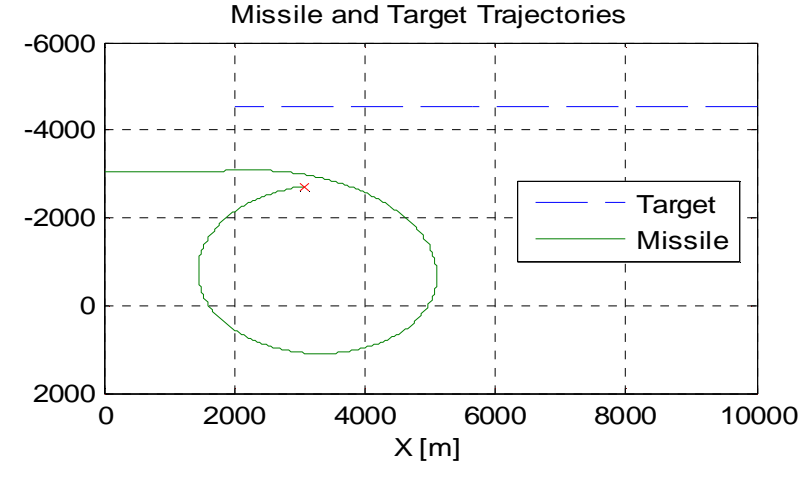

Fig. 30 Missile and target trajectory in presence of MBJ without ECCM circuit 


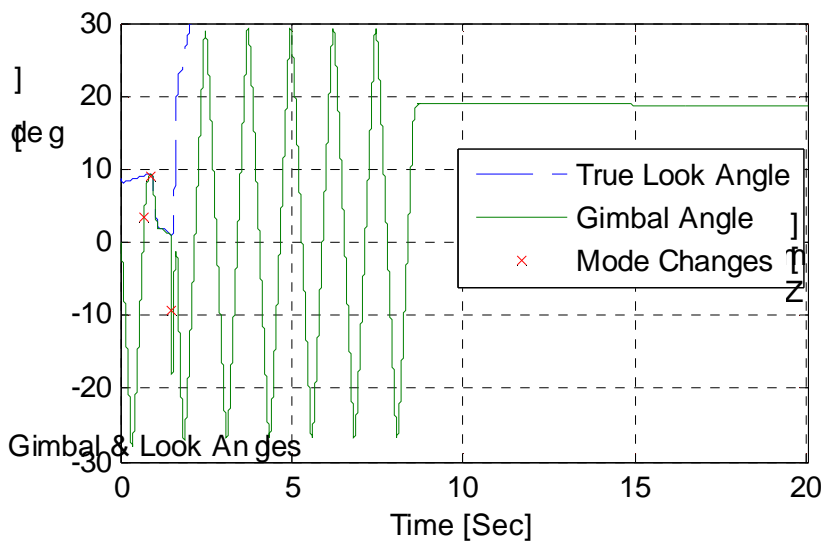

Fig. 31 Gimbal and look in presence MBJ without ECCM circuit

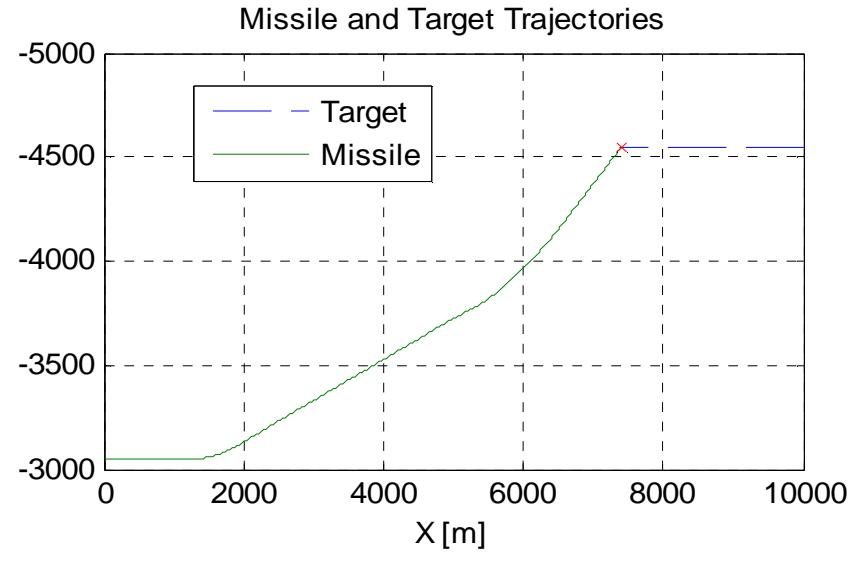

Fig. 32 Missile and target trajectory in presence of a uniform noise with ECCM circuit

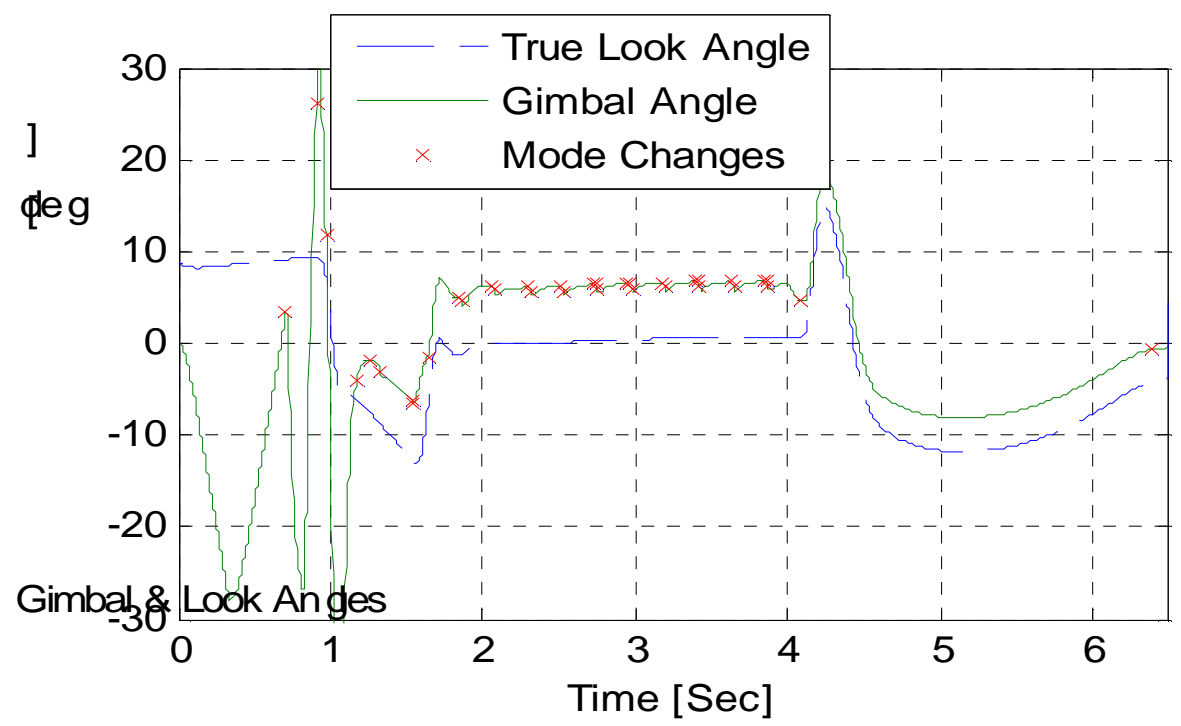

Fig. 33 Gimbal and Look angle in presence of a uniform noise with ECCM circuit 
Table. 2 The miss distances in both cases (maneuvering and non maneuvering target)

\begin{tabular}{|c|c|c|c|c|c|c|}
\hline Source & BJ & MBJ & $\begin{array}{c}\text { BJ+ Ga. } \\
\text { noise }\end{array}$ & $\begin{array}{c}\text { MBJ+ Ga. } \\
\text { noise }\end{array}$ & $\begin{array}{c}\text { BJ+ uniform } \\
\text { noise }\end{array}$ & $\begin{array}{c}\text { MBJ+ uniform } \\
\text { noise }\end{array}$ \\
\hline $\begin{array}{c}\text { Non man. Target miss } \\
\text { distance(m) }\end{array}$ & 4.1 & 6.2 & 8 & Missile miss & 11 & 20 \\
\hline $\begin{array}{c}\text { Maneuvering target miss- } \\
\text { distance(m) }\end{array}$ & 17.7 & 10.2 & 22 & Missile miss & 38. & 34 \\
\hline
\end{tabular}

\section{Conclusions:}

This paper addressed the sources of uncertainties that challenge a radio seeker for semi-active homing missile guidance and control system. A deep investigation for typical radio seeker is developed associated with a seeker modeling. The ECM techniques, noise and target maneuvers are among the uncertainties. An ECCM circuit is proposed for degrading the significant effect of both BJ and MBJ techniques special in presence of both noise and random target maneuvers. An integrated seeker model associated with the proposed ECCM circuit modeling for semi-active homing missile system is carried out. Miss distance and missile dynamic response are the performance measure. An adequate and promising response for the proposed circuitry is justified. Yet more research is under way for improving the seeker performance to cope with various uncertainties.

\section{References:}

[1].Bou-Karda Nader W. "Modern Integrated Electronic Warfare Systems and Their Performance Analysis", M.sc. Thesis, M.T.C, Egypt, 1998.

[2] Leroy B. Van Brunt, "Applied ECM', Vol.”, EW Engineering Inc. First Edition, U.S.A, 1995.

[3] S.L .Johnston "Radar electronic counter countermeasures," IEEE Transaction on aerospace and electronic systems vo.1. Jan 1978.

[4] J.V. Difranco and C.Kaiteris, "Radar reference receiver in clear and jamming environment", IEEE Transaction on aerospace and electronic systems Vol. 5 September 1978.

[5] Rashid .N.R. "Analysis of Radar performance under jamming" M.sc. thesis. Egypt 1994.

[6] W.V. Andrew, "The effects of Multi-path propagation on Low-Altitude Detect Pulsed-Doppler RADAR Systems, December 1990, Arizona State University.

[7] ELsese, A.E.H. "Using Phase-Front Distortion to deceive the homing missiles", MTC. 2000.

[8] Sherman S.M., "Monopulse Principles and Techniques". Artech House, 1984.

[9].Menesy M.A., "Evaluation of the CW-Doppler Seeker of Semi-active Tactical Missiles in the Presence of ECM", M.s.c. Thesis, Military Technical College, Egypt, 1992.

[10].Blanch aid A., "Interference in Phase Lock Loops" IEEE Trans. Aerospace\& Electronic Systems, Vol. AES-10, September 1974.

[11] Wael.M.A, "Investigating the Performance of Radio Seekers and ECCM. M.sc. Thesis, M.T.C, Egypt, 2001.

[12] Zarchan P.'Tactical and Strategic Missile Guidance”, American Institute of Aeronautics, Inc .Second Edition, 1994.

[13] Lin C.F, "Modern Navigation, Guidance, and Control Processing", American GNC Corporation. 1991. 